\title{
A diagnostic scale for Alzheimer's disease based on cerebrospinal fluid biomarker profiles
}

\author{
Sylvain Lehmann ${ }^{*}$, Julien Dumurgier ${ }^{2}$, Susanna Schraen ${ }^{3}$, David Wallon ${ }^{4}$, Frédéric Blanc ${ }^{5,6}$, Eloi Magnin ${ }^{7}$, \\ Stéphanie Bombois ${ }^{8}$, Olivier Bousiges ${ }^{5,9,13}$, Dominique Campion ${ }^{4}$, Benjamin Cretin ${ }^{5}$, Constance Delaby ${ }^{1}$, \\ Didier Hannequin ${ }^{4}$, Barbara Jung ${ }^{5}$, Jacques Hugon ${ }^{2}$, Jean-Louis Laplanche ${ }^{10}$, Carole Miguet-Alfonsi ${ }^{11}$, \\ Katell Peoc' $h^{10}$, Nathalie Philippi ${ }^{5,6}$, Muriel Quillard-Muraine ${ }^{4}$, Bernard Sablonnière ${ }^{3}$, Jacques Touchon ${ }^{12}$, \\ Olivier Vercruysse ${ }^{3}$, Claire Paquet ${ }^{2}$, Florence Pasquier ${ }^{8}$ and Audrey Gabelle $e^{1,12}$
}

\begin{abstract}
Introduction: The relevance of the cerebrospinal fluid (CSF) biomarkers for the diagnosis of Alzheimer's disease (AD) and related disorders is clearly established. However, the question remains on how to use these data, which are often heterogeneous (not all biomarkers being pathologic). The objective of this study is to propose to physicians in memory clinics a biologic scale of probabilities that the patient with cognitive impairments has an Alzheimer's disease (AD) pathologic process.

Methods: For that purpose, we took advantage of the multicenter data of our Paris-North, Lille, and Montpellier (PLM) study, which has emerged through the initial sharing of information from these memory centers. Different models combining the CSF levels of amyloid- $\beta 42$, tau, and p-tau(181) were tested to generate categories of patients with very low $(<10 \%)$, low $(<25 \%)$, high $(>75 \%)$, and very high predictive values $(>90 \%)$ for positive AD. In total, 1,273 patients (646 AD and 627 non-AD) from six independent memory-clinic cohorts were included.

Results: A prediction model based on logistic regressions achieved a very good stratification of the population but had the disadvantages of needing mathematical optimization and being difficult to use in daily clinical practice. Remarkably, a simple and intuitive model based on the number (from zero to three) of three pathologic CSF biomarkers resulted in a very efficient predictive scale for $\mathrm{AD}$ in patients seen in memory clinics. The scale's overall predictive value for AD for the different categories were as follows: class 0, 9.6\% (95\% confidence interval (Cl), 6.0\% to 13.2\%); class 1, 24.7\% (95\% Cl, $18.0 \%$ to 31.3\%); class 2, 77.2\% (95\% Cl, 67.8\% to 86.5\%); and class 3, 94.2\% (95\% Cl, $90.7 \%$ to $97.7 \%$ ). In addition, with this scale, significantly more patients were correctly classified than with the logistic regression. Its superiority in model performance was validated by the computation of the net reclassification index (NRI). The model was also validated in an independent multicenter dataset of 408 patients (213 AD and 195 non-AD).
\end{abstract}

Conclusions: In conclusion, we defined a new scale that could be used to facilitate the interpretation and routine use of multivariate CSF data, as well as helping the stratification of patients in clinical research trials.

\section{Introduction}

Intense research efforts have been conducted to develop and validate biomarkers to predict, detect, and follow up the progression of the disease or impact of potential new disease-modifying treatments of Alzheimer's disease (AD). Brain imaging and biologic biomarkers are now included

\footnotetext{
* Correspondence: s-lehmann@chu-montpellier.fr

${ }^{1} \mathrm{CHU}$ de Montpellier and Université Montpellier I, IRMB, CCBHM, Laboratoire de Biochimie Protéomique Clinique, 80 Avenue Augustin Fliche, 34295 Montpellier, France

Full list of author information is available at the end of the article
}

in the recommended $\mathrm{AD}$ diagnostic criteria [1-3]. Various scales or tools are available to physicians involved in $\mathrm{AD}$ research for better interpreting the positive or negative results of biomarkers for establishing a clinical diagnosis, such as the detection of hippocampal atrophy with the Scheltens' scale [4] and the positivity of amyloid load on functional brain imaging via the Jack's visual scale [5].

Although biologic cerebrospinal fluid (CSF) biomarkers are among the most studied and validated in clinical practice [6-8], no sort of "visual" scale concerning 
biologic biomarkers is available. Numerous studies did indeed show that $A D$ patients display characteristic CSF changes with decreased levels of $\beta$-amyloid1-42 (A $\beta 42$ ) and elevated levels of total tau protein (tau) and its phosphorylated form at threonine 181 (p-tau) $[7,9,10]$. Interestingly, these biomarkers are directly related to neuropathologic changes [11] present in the disease. Therefore, one would expect that the presence of these three biomarkers would be highly indicative of $\mathrm{AD}$, whereas their absence would strongly disqualify this diagnosis. Other biomarkers represented by isoforms of $A \beta(A \beta 40, A \beta 38)$ or linked to oxidative stress and inflammation $[12,13]$ may also contribute to the diagnosis of $\mathrm{AD}$, in particular when combined with tau or $\mathrm{A} \beta 42$. Apart from familial forms of $\mathrm{AD}$, genetic profiles [14], in particular apolipoprotein $\mathrm{E}$ status, which represents the most prominent risk factor, is not yet used as a diagnostic tool.

So, today we can only rely routinely on the commonly measured CSF biomarkers $A \beta 42$, tau, and $p$-tau to help us with $\mathrm{AD}$ diagnosis. In clinical practice, after control of preanalytic biases [15-17] and standardization of procedures [18-20], the performance of CSF biomarkers is satisfactory with a coupled sensitivity/specificity as high as $80 \%$ when used alone or combined. The combination of these biomarkers increases their performance, as demonstrated for $A \beta 42$ and tau [21] or $A \beta 42$ and p-tau [22,23]. In most $\mathrm{AD}$ biomarker studies, results are represented by the best sensitivity/specificity and area under the ROC curves (AUC). It is, however, difficult to use this information directly when biomarkers are being used in routine practice to help physicians with $\mathrm{AD}$ diagnosis [6-8]. We need a way to convert the variation of the three biomarkers into a probability scale for AD. A leading study from Spies et al. [24] proposed a logistic regression of logarithmic-transformed values of $A \beta 42$ and p-tau values and sex defined classes of $\mathrm{AD}$ probability. Logistic regressions already demonstrated their relevance in differentiating $\mathrm{AD}$ patients from non- $\mathrm{AD}$ patients $[7,23]$.

In the present study, we conducted an evaluation, based on multicenter data from our PLM cohort [25], of a simpler scale based solely on the numbers (from 0 to 3 ) of pathologic biomarkers (A $\beta 42$, tau, and $\mathrm{p}$-tau) identified. This intuitive scale was very efficient for generating categories of patients seen in memory clinics with a refined probability of $\mathrm{AD}$, and it will represent a valuable tool for facilitating the interpretation and routine use of multivariate CSF data for stratifying patients into clinical research trials.

\section{Material and methods}

\section{Study design and subjects}

Patients $(1,273)$ who had a lumbar puncture were recruited between January 2008 and December 2011 from the three initial PLM centers (Paris-North, Lille, and Montpellier) specialized in the care management of patients with cognitive disorders (Table 1). These centers used the same diagnostic procedures and criteria [25]. All patients had a thorough clinical examination, including biologic laboratory tests, neuropsychological evaluations, and brain imaging. Patients were classified into two groups: $\mathrm{AD}$ (as defined by the NINCDS-ADRDA criteria [26]), and non-AD (NAD) patients. NAD diagnosis (that is, frontotemporal lobar degeneration, semantic dementia, Lewy body and Parkinson diseases, progressive supranuclear palsy, amyotrophic lateral sclerosis, normal-pressure hydrocephalus, and psychiatric disorders), were defined by the commonly validated international criteria. Important for this study, the used diagnostic criteria for AD do not include CSF biomarkers (otherwise, that would bias the evaluation of the interest of the scale, which was based on biomarker values). Mild cognitive impairment, as well as phenotypes that were mixed with $\mathrm{AD}$ or might correspond to specific/early forms of $\mathrm{AD}$, were excluded from the cohorts (mixed dementia, primary progressive aphasia, amyloid angiopathy). CSF was collected by using standardized collection, centrifugation, and storage conditions in different centers [25].

A second set of data from 408 patients with the same clinical characteristics was generated from the three new PLM centers (Rouen, Strasbourg, and Besançon) also specialized in the care management of patients with cognitive disorders.

\section{CSF samples and assays}

CSF A $\beta 42$, tau, and p-tau concentrations were measured by using standardized commercially available INNOTEST sandwich ELISA, according to the manufacturer's procedures (Fujirebio Europe NV, formerly Innogenetics NV). As the data were generated in the different centers through the routine activity of their laboratories, the lots of assay kits were variable within and in between laboratories. The quality of the results was ensured by the use of validated standard operating procedures and internal quality controls $(\mathrm{QCs})$. The range of the $\mathrm{QC}$ coefficient of variation for $\mathrm{A} \beta 42$ across the different lots for the six laboratories was $5 \%$ to $11 \%$. For tau and p-tau, the range was $8 \%$ to $14 \%$ and $6 \%$ to $14 \%$, respectively. The use of external QC ensured also the quality of the results and the validity of the intersite data comparison [19]. The Paris, Lille, and Montpellier centers contributed with two sets of data labeled "-1" or "-2" generated from different collection tubes (for the -1 cohort in Montpellier, Greiner, catalog number 1882 81; in Lille, Becton Dickinson, catalog number Falcon 35 2097; in Paris, CML, catalog number TC10PCS, and for the -2 cohorts: Sarstedt, catalog number 62.610.201) [20]. These two sets of data differed in their optimal cutoffs $(\leq 506$ and $\leq 834$ for A $\beta 42 ;>343$ and $>340$ for tau; $>64$ and $>62$ for p-tau; see Additional file 1) in relation with the preanalytic properties of the tube that affected mostly A $\beta 42$ levels [27]. 
Table 1 Population demography and biomarker values

\begin{tabular}{|c|c|c|c|c|c|c|c|c|c|c|c|c|c|c|c|c|c|c|c|c|}
\hline \multicolumn{3}{|c|}{ Paris-1 } & \multicolumn{3}{|c|}{ Paris-2 } & \multicolumn{3}{|c|}{ Lille-1 } & \multicolumn{3}{|c|}{ Lille-2 } & \multicolumn{3}{|c|}{ Montpellier-1 } & \multicolumn{3}{|c|}{ Montpellier-2 } & \multicolumn{3}{|c|}{ RSB } \\
\hline $\mathrm{AD} n=118$ & Mean & $\overline{S D}$ & $A D n=41$ & Mean & $\overline{S D}$ & $A D n=118$ & Mean & $\overline{S D}$ & $A D n=73$ & Mean & $\overline{S D}$ & $A D n=129$ & Mean & $\overline{S D}$ & $A D n=142$ & Mean & $\overline{S D}$ & $A D n=213$ & Mean & $\overline{S D}$ \\
\hline Age & 73.6 & 8.8 & Age & 70.9 & 8.9 & Age & 68.3 & 9 & Age & 67 & 9.5 & Age & 69.7 & 8.8 & Age & 71.1 & 10.1 & Age & 66.3 & 8.7 \\
\hline$A \beta 42$ & 440 & 189 & $A \beta 42$ & 594 & 238 & $A \beta 42$ & 338 & 162 & $A \beta 42$ & 603 & 245 & $A \beta 42$ & 505 & 224 & $A \beta 42$ & 654 & 256 & $A \beta 42$ & 420 & 224 \\
\hline Tau & 598 & 295 & Tau & 543 & 279 & Tau & 608 & 336 & Tau & 778 & 364 & Tau & 611 & 327 & Tau & 702 & 727 & Tau & 666 & 407 \\
\hline p-tau & 99 & 40.4 & p-tau & 86 & 34 & p-tau & 98.1 & 46.9 & p-tau & 101.5 & 41.2 & p-tau & 85.9 & 40.2 & p-tau & 86 & 37.7 & p-tau & 92.4 & 38.1 \\
\hline MMSE & 19.4 & 5.6 & MMSE & 18.7 & 6.7 & MMSE & 18.1 & 6.5 & MMSE & 19.6 & 6.3 & MMSE & 21.9 & 5.5 & MMSE & 20.7 & 7.4 & MMSE & 19.0 & 6.1 \\
\hline Sex $(\% M)$ & $47 \%$ & & $\operatorname{sex}(\% M)$ & $65 \%$ & & sex $(\% M)$ & $37 \%$ & & sex (\%M) & $47 \%$ & & sex $(\% M)$ & $47 \%$ & & sex $(\% M)$ & $49 \%$ & & $\operatorname{sex}(\% M)$ & $50 \%$ & \\
\hline NAD $n=53$ & Mean & SD & NAD $n=68$ & Mean & $\mathrm{SD}$ & NAD $n=128$ & Mean & SD & NAD $n=51$ & Mean & SD & NAD $n=215$ & Mean & SD & NAD $n=147$ & Mean & SD & NAD $n=195$ & Mean & SD \\
\hline Age & 62.1 & 13.1 & Age & 67.4 & 11.1 & Age & 67.3 & 10.7 & Age & 64.6 & 10.7 & Age & 64.1 & 13.6 & Age & 63.4 & 13.6 & Age & 65.4 & 10.1 \\
\hline$A \beta 42$ & 686 & 243 & $A \beta 42$ & 843 & 246 & $A \beta 42$ & 494 & 192 & $A \beta 42$ & 974 & 355 & $A \beta 42$ & 706 & 266 & $A \beta 42$ & 999 & 373 & $A \beta 42$ & 723 & 346 \\
\hline Tau & 253 & 226 & Tau & 223 & 141 & Tau & 273 & 197 & Tau & 284 & 149 & Tau & 291 & 233 & Tau & 310 & 241 & Tau & 339 & 258 \\
\hline p-tau & 48.6 & 23.1 & p-tau & 43.5 & 19.3 & p-tau & 52.6 & 28.7 & p-tau & 46.5 & 15.8 & p-tau & 44.8 & 23.7 & p-tau & 38.4 & 18.6 & p-tau & 49.3 & 25.8 \\
\hline MMSE & 23 & 5.3 & MMSE & 23.6 & 4.9 & MMSE & 21.3 & 5.5 & MMSE & 21.2 & 6 & MMSE & 20.7 & 7.1 & MMSE & 21.1 & 6.9 & MMSE & 21.0 & 6.1 \\
\hline Sex (\%M) & $53 \%$ & & Sex (\%M) & $49 \%$ & & sex (\%M) & $51 \%$ & & sex $(\% M)$ & $59 \%$ & & sex (\%M) & $53 \%$ & & sex (\%M) & $55 \%$ & & sex (\%M) & $51 \%$ & \\
\hline
\end{tabular}

Mean and standard deviation of demographic data, CSF biomarker levels (Aß42, tau, and p-tau) and Mini Mental Score (MMSE) for the patients with AD or NAD diagnosis in the different centers (Montpellier, Lille, Paris) in two independent cohorts that differed by their collection tubes $(-1,-2)$ and in the three additional PLM centers: Rouen, Strasbourg, and Besançon (RSB). 
We pooled the data from the three additional PLM centers (Rouen, Strasbourg, and Besançon), which used standard cutoff biomarker values in relation to the type of collection tube used [20]. The lumbar puncture, the CSF biomarkers measurement, and the clinical examination (including the diagnosis) were part of standard care. This observational study on routine biologic analyses is not considered in France to be "biomedical research," and it does not necessitate informed consent or ethical approval. Authorization for handling personal data has been granted by the French Data Protection Authority (CNIL) under the number $1709743 \mathrm{v0}$.

\section{Statistical analysis}

Statistical analyses were computed with the MedCalc software (11.3). The logistic regression was performed by following a method similar to that of Spies et al. [24]. In brief, log-transformed CSF biomarkers and sex were entered with backward stepwise selection by using a significance level of 0.10 . The logistic regression generates the coefficients of a formula to predict the logistic transformation of the probable presence of $\mathrm{AD}(\mathrm{p}(\mathrm{AD}))$ as follows:

$\mathrm{p}(\mathrm{AD})=1 /(1+1 \mathrm{e}-[$ intercept + score $])$

where score $=$ coefficient $\times \ln (\mathrm{A} \beta 42)+$ coefficient $\times \ln$ $(\mathrm{p}$-tau $)+$ coefficient $\times$ Sex. Probability classes to discriminate between $\mathrm{AD}$ and non-AD patients were obtained by selecting the following ranges of $\mathrm{p}(\mathrm{AD})$ : class 0,0 to 0.1 ; class 1 , 0.1 to 0.5 ; class 2, 0.5 to 0.9 ; and class $3,0.9$ to 1.0 .

We did not perform the logistic regression analysis in one training population with the goal to apply the resulting classification to the other populations, but we rather recalculated the coefficients in each cohort to generate best-fitted models. Receiver operating characteristic (ROC) curves were used to represent sensitivity and specificity for AD detection. ROCs are generated from continuous diagnostic variables. The CSF biomarkers and their ratio are continuous, as well as the $\mathrm{p}(\mathrm{AD})$ values obtained after the logistic regression. Regarding the scales, we plotted the curve by using the four values $0,1,2$, and 3 applied to the different samples.

To compare the classifications of patients, we used the net reclassification index (NRI) [28]. The NRI is based on reclassification constructed separately for participants with and without the event of interest (that is, AD or non-AD diagnosis), and quantifies the correct movement into classes, upward for events and downward for nonevents. At first, the following probabilities were calculated: p(up_AD) $=$ (number of cases in which the class was moving up between two classifications of AD patients)/ (number of AD patients); $\mathrm{p}($ down_AD $)=($ number of cases in which the class was moving down between two classifications of $\mathrm{AD}$ patients)/(number of $\mathrm{AD}$ patients); $\mathrm{p}($ up_NAD $)=($ number of cases in which the class was moving up between two classifications of NAD patients)/ (number of NAD patients); $\mathrm{p}($ down_NAD) $=$ (number of cases in which the class was moving down between two classifications of NAD patients)/(number of NAD patients). We assumed that correctly classifying an $\mathrm{AD}$ patient was as important as correctly classifying an NAD patient, and therefore we computed the NRI by using the formula: NRI = (p(up_AD)-p(down_AD) $)-(p($ up_NAD)-p(down_NAD $))$.

\section{Results}

Demographics and biomarker values for the different cohorts are presented in Table 1. As expected, differences in individual biomarker concentrations between $\mathrm{AD}$ and NAD were apparent across all cohorts. ROC curves for A $\beta 42$, tau, and p-tau were used to compute AUCs (Table 2) and optimal cutoff values (Additional file 1). AUCs were also calculated for $A \beta 42 /$ tau and $A \beta 42 / p$-tau ratios, as well as the $A \beta /$ tau index (IATI) [21] (Table 2). The Paris, Lille, and Montpellier PLM centers provided data from two independent cohorts $(-1$ and -2$)$ that differed by the type of collection tube used (see Material and methods section). This explains the variations in cutoff values; the second tube having been selected for its low $A \beta 42$ absorption [27], therefore resulting in higher cutoff values [20]. All patients of these three centers for whom samples were collected in tube 1 or 2 were also differentiated into two additional populations called PLM-1 and PLM-2. This was one way to evaluate the $\mathrm{AD}$ scale on multicenter cohorts after standardization of biologic and clinical practices. Data from these populations were used to compare predictions based on a logistic regression performed as shown by Spies et al. [24]. Interestingly, sex was never retained in the model used for our cohorts (Additional file 1). The models were very efficient in differentiating AD from nonAD (NAD) patients for all cohorts with AUCs close to 0.9 in most cases (Table 2).

The distribution of $\mathrm{AD}$ and NAD patients was then evaluated according to four classes: between $\mathrm{p}(\mathrm{AD}) 0$ to 0.1 for class 0 ; 0.1 to 0.5 for class $1 ; 0.5$ to 0.9 for class 2 ; and 0.9 to 1 for class 3 (Figure 1A,B and Additional file 2). The percentage of $\mathrm{AD}$ in each class was also computed (Figure 1C). As expected, in class 0, we found only around $10 \%$ of $\mathrm{AD}$ patients, whereas in class 3 , this number was close to $90 \%$, with slight variations related to differences in AD prevalence in these populations (Table 1).

The PLM scale composed of four classes was then designed, based on a very simple and intuitive rule: class 0 , corresponding to no pathologic biomarkers (below cutoff for A $\beta 42$, above cutoff for tau and p-tau); class 1 , corresponding to one pathologic biomarker of three; class 2, corresponding to two pathologic biomarkers of three; and class 3, with all three biomarkers being pathologic. We calculated the sensitivity and specificity of this scale, with classes 0 and 1 grouped on one side, and classes 2 and 3, on the other side for NAD and AD diagnoses, 
Table 2 AUCs

\begin{tabular}{cccccccccc}
\hline AUC & Paris-1 & Paris-2 & Lille-1 & Lille-2 & Mtp-1 & Mtp-2 & PLM-1 & PLM-2 & Mean \\
\hline A $\boldsymbol{B 4 2}$ & 0.81 & 0.768 & 0.778 & 0.826 & 0.747 & 0.778 & 0.772 & 0.787 & 0.783 \\
Tau & 0.898 & 0.921 & 0.869 & 0.905 & 0.84 & 0.852 & 0.86 & 0.88 & 0.878 \\
p-tau & 0.911 & 0.913 & 0.87 & 0.917 & 0.842 & 0.91 & 0.875 & 0.912 & 0.894 \\
IATI & 0.902 & 0.895 & 0.858 & 0.917 & 0.827 & 0.877 & 0.855 & 0.896 & 0.878 \\
A $342 /$ tau & 0.913 & 0.921 & 0.88 & 0.927 & 0.849 & 0.882 & 0.874 & 0.905 & 0.894 \\
A 3 42/p-tau & 0.923 & 0.92 & 0.875 & 0.92 & 0.86 & 0.924 & 0.884 & 0.924 & 0.904 \\
Logi. reg. & 0.926 & 0.928 & 0.892 & 0.936 & 0.869 & 0.933 & 0.896 & 0.932 & 0.914 \\
Log. reg. scale & 0.917 & 0.931 & 0.872 & 0.927 & 0.838 & 0.918 & 0.876 & 0.919 & 0.900 \\
PLM scale & 0.94 & 0.931 & 0.887 & 0.919 & 0.863 & 0.933 & 0.883 & 0.924 & 0.910 \\
\hline
\end{tabular}

Area under the ROC for the individual biomarkers $A \beta 42$, tau and $p$-tau, the IATI [21] and the ratios $A \beta 42 / \operatorname{tau}$ and $A \beta 42 / p$-tau. The values of the $p(A D)$ obtained after logistic regression were also used to calculate the AUC. The logistic regression values were separated into four classes ( 0 to 3$)$, which values were then used, just as for the PLM scale, to calculate the AUCs.

respectively. Hence, a sample was defined as positive for $\mathrm{AD}$ if two or three biomarkers were pathologic (class 2 or 3 ). The performance of this simple rule was very high, as demonstrated by the sensitivity and specificity reached across the different cohorts (see Additional file 2). Moreover and interestingly, when all AUCs were compared (Table 2), the PLM scale was second best after the logistic regression.
Distributions of $\mathrm{AD}$ and NAD patients, as well as percentage of $\mathrm{AD}$ patients in each class, were then computed and plotted for the PLM scale (Figure 1A-C; Additional file 2). The percentage of $\mathrm{AD}$ patients in each class, which actually corresponded to the positive predictive value (PPV), that is, the percentage of true positives among all samples in the class, was compared between the two prediction models (Figure 1C). Predictive values were not significantly different
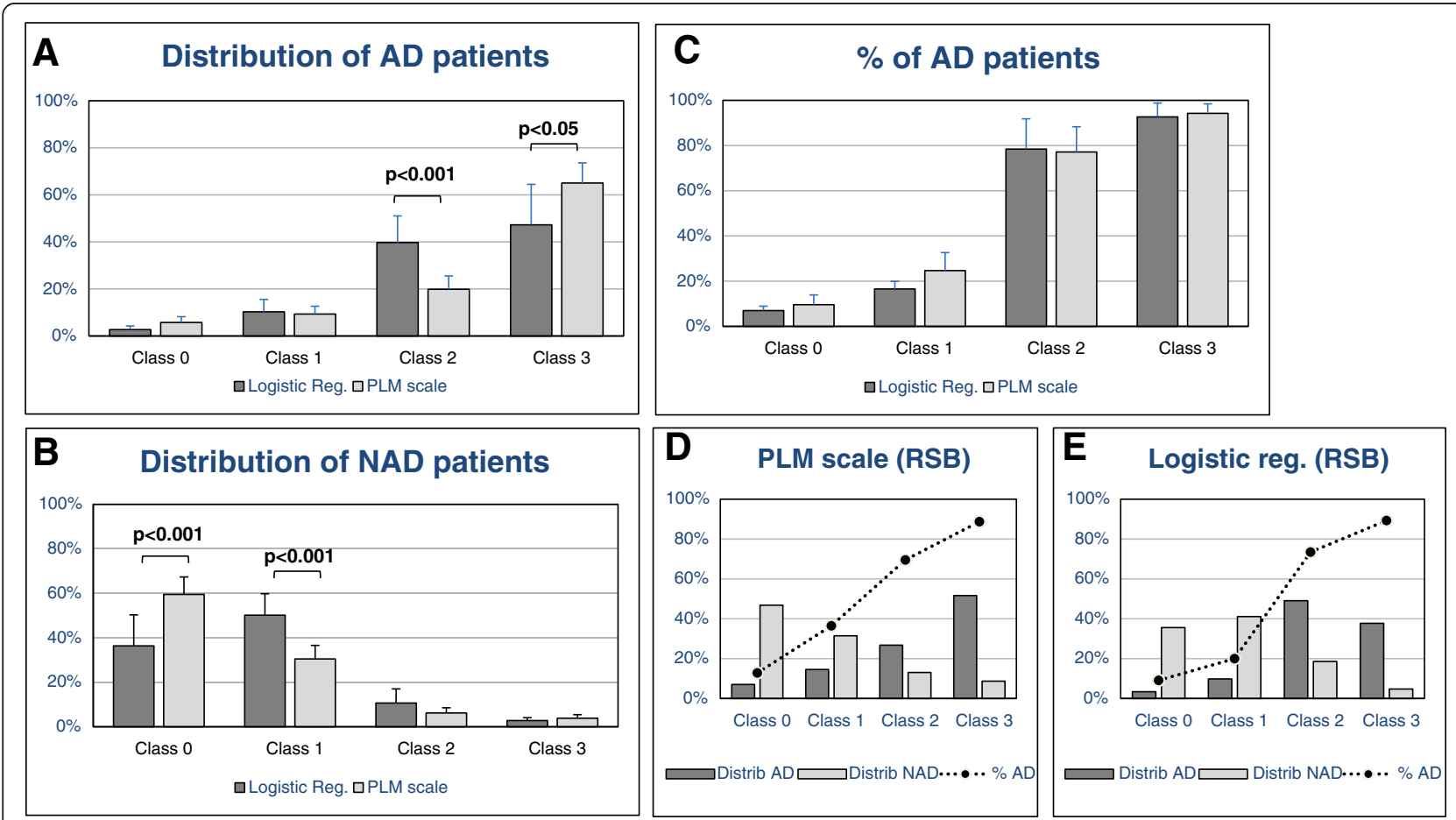

Figure 1 Distribution and percentage of the AD and NAD patients between the different classes. (A, B) The mean ( \pm SD) was plotted of the distribution of the AD (A) and NAD (B) patients in the Paris, Lille, and Montpellier cohorts and across the four classes based on the logistic regression or the PLM scale. Significant differences (Student $t$ test) were found between the percentage of AD patients in classes 2 and 3 (A), as well as between the percentage of NAD patients in classes 0 and 1 (B). (C) The mean $( \pm S D)$ of the percentage of AD patients in each class was plotted. No significant difference was apparent. (D, E) The distribution of AD (dark gray bars) and NAD (light gray bars) patients in the populations of the Rouen, Strasbourg, and Besançon (RSB) centers. The percentage of AD in each class is also plotted (black dots linked a dotted line). Data obtained by using the PLM scale (C) show more AD patients in class 3 and NAD in class 0 than for the logistic regression (E). 
between the logistic regression and PLM scale (Figure 1C). For the PLM scale, the average predictive values for $\mathrm{AD}$ in these cohorts were for class $0,9.6 \%$ (95\% CI, $6.0 \%$ to $13.2 \%$ ); for class 1: $24.7 \%$ (95\% CI, $18.0 \%$ to $31.3 \%$ ), for class 2 : $77.2 \%$ (95\% CI, $67.8 \%$ to $86.5 \%$ ), and for class 3, $94.2 \%$ (95\% CI, $90.7 \%$ to $97.7 \%$ ) (Figure 2).

The difference in patient distribution between the two prediction models was, however, significantly different, as the PLM scale had more AD patients in class 3 as well as more NAD patients in class 0 compared with the prediction obtained with the logistic regression (Figure 1A, B). This distribution of AD and NAD patients in different classes was also evaluated in an additional dataset from Rouen Strasbourg and Besançon (RSB) centers (Table 1). We used the standard cutoffs and logistic regression coefficients (Additional file 2) to compute and represent in Figure 1 (panels D and E) the distribution of AD and NAD patients across the different classes, as well as the percentage of AD patients in each class. With the PLM scale, profiles were comparable to those obtained before, with a better distribution of patients (more AD patients in class 3 , and more NAD patients in class 0 ) and similar percentages of $\mathrm{AD}$ patients in each class.

To compare the classification resulting from the logistic regression and the PLM scales, we calculated the NRI (see Material and methods section) on the PLM-2 population. We observed that the PLM scale resulted in 23.25\% more patients better classified (that is, having a higher scale value in the case of $\mathrm{AD}$ patients, and a lower value for nonAD patients). Similar results were obtained when the NRI was applied to the different cohorts (data not shown).

\section{Discussion}

Diagnostic measures of $\mathrm{AD}$ include clinical observation, assessment of cognitive functions, including memory testing as well as advanced neuroimaging examinations such as magnetic resonance imaging (MRI) or positron emission tomography (PET), and CSF biomarkers [29]. In the present study, we validated the relevance of CSF biomarkers when used individually or within ratios [7,21-23]. Identifying one or several biomarkers or ratios as pathological is clinically relevant for the diagnosis of $\mathrm{AD}$. However, the question remains as to how to use this element, which is often heterogeneous (not all biomarkers being pathological) in clinical practice. This complexity renders problematic the interpretation of biomarker results, and it differs between practitioners or centers.

Different rating systems or scales have being designed to help clinicians evaluate dementia. The clinical dementia rating (CDR), which is a 5-point scale based on cognitive and functional performances, does provide valuable clinical information [30]. The medial temporal lobe atrophy visual rating scale introduced by Scheltens et al. [31] is also a good example of a biomarker scale facilitating the diagnosis

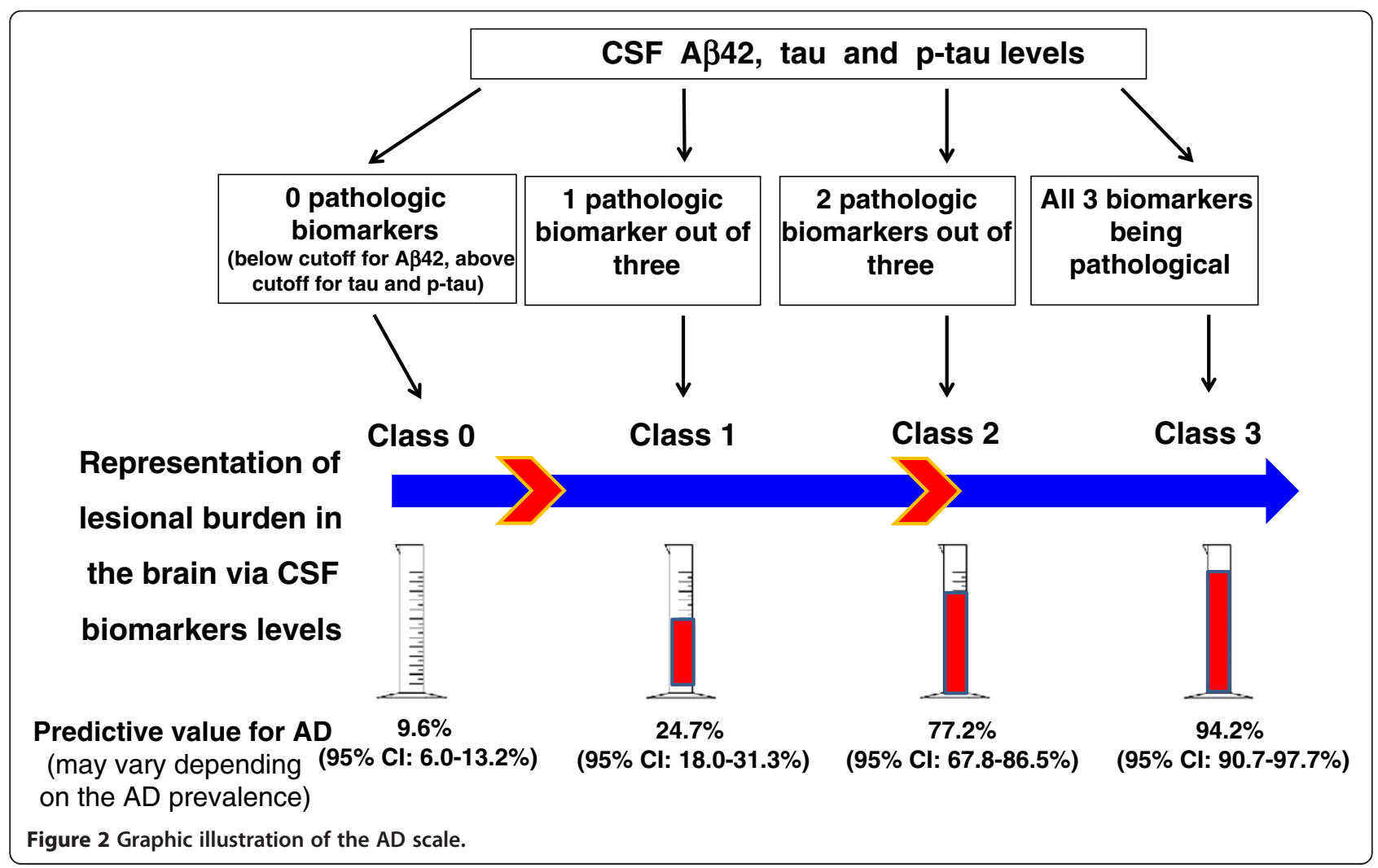


of $\mathrm{AD}$. The challenge is to transform a multivariate panel into a score that predicts clinical outcomes. This was recently done to predict the conversion of mild cognitive impairment (MCI) patients into AD by using CSF Aß42, MRI, and PET data [32]. However, this model cannot be applied to our paradigm, which focuses on memory clinic cohorts suspected of having AD or other differential dementia diagnoses. Very relevant in this context is the leading work of Spies et al., [24] who used a logistic regression on CSF biomarkers and sex to define a predictive model for $\mathrm{AD}$. This model reached the highest performance for the diagnosis of $\mathrm{AD}$, and it provides a continuous predictive scale made of 10 classes of $\mathrm{AD}$ probability (that is, none to $10 \%, 10 \%$ to $20 \%$ ).

When we reproduced this approach for our datasets, the logistic regression did not retain sex in the models. The higher percentage of women in the AD population in the Spies cohort was probably responsible for this difference. In any case, the performance of the logistic regression was outstanding, as it resulted in the highest AUCs among individual or biomarker ratios (Table 2). To match the reports already used in clinical practice, we wanted to generate a simple rating with classes bearing very low $(<10 \%)$, low $(<25 \%)$, high $(>75 \%)$, and very high predictive values $(>90 \%)$ for $\mathrm{AD}$. Four logistic regression classes with $\mathrm{p}(\mathrm{AD})$ ranging from 0 to 0.1 , 0.1 to $0.5,0.5$ to 0.9 , and 0.9 to 1 were therefore generated. This resulted in classes having an expected percentage of $\mathrm{AD}$ prediction (Figure 1C). We then compared this logistic regression approach with an intuitive classification based solely on the numbers (from 0 to 3 ) of pathological CSF biomarkers (Aß42, tau, and p-tau) (Figure 2).

The performance of this simple scale was evaluated in the different cohorts by using as a criterion of $\mathrm{AD}$ the presence of two or three pathologic biomarkers. Surprisingly, this simple rule resulted in high sensibility/ specificity/AUCs. The predictive values of this "PLM scale" for the different classes were also similar to the ones obtained with the logistic regression (Figure 1C). Differences in the distribution of $\mathrm{AD}$ and NAD patients between classes were, however, noticeable, with significantly more patients correctly classified with the PLM scale. This apparent superiority of the PLM scale was validated by computing the NRI, which is a statistical tool designed to assess improvement in model performance offered by a new classification. This result could be explained by the "discontinuous" nature of the PLM scale, which is better suited to the four probability classes that we had targeted. Of note, a discrepancy between predicted and observed percentage of $\mathrm{AD}$ in regression classes was already observed by Spies et al. [24]. This triggered these authors to generate a second model with an extensive correction based on a second regression. This fine-tuning might overfit the models based on the individuals within a cohort, and it is therefore not adapted for the use of the scale in a predictive way for the classification of new patients in memory clinics.

In any case, the PLM scale outperformed the logistic regression and has the advantage of being used without complex mathematical adjustments. In addition, this classification gave a direct access to the percentage of discarding profiles, that is, $\mathrm{AD}$ patients with none of the biomarkers being positive (class 0 ), and conversely, NAD patients with three biomarkers being positive (class 3).

One of our study's limits resides in using as reference the final clinical diagnosis, which, in the absence of neuropathologic information, can sometimes remain uncertain. However, diagnoses were established by experienced multidisciplinary teams based on clinical, neuropsychological, and imaging data. The cross-validation of the results in the different cohorts also guarantees the reliability and relevance of our conclusions. Of note, the scale relies on the use of biomarker cutoffs that might differ in the different centers. The continuous improvement of the quality of the assays, thanks to the use of quality control, and the homogenization of preanalytics and operating procedure [15-20] may result in a scale that will use "unified" cutoffs. It is important to underline that the predictive value of the PLM scale is valid only to evaluate whether memory impairments or dementia is due to $\mathrm{AD}$ for patients seen in memory clinics. In other situations, and in particular for assessing the conversion from $\mathrm{MCI}$ to $\mathrm{AD}$, the design and/ or predictive value of the PLM scale should be revaluated.

We also must keep in mind that if we expect the distribution of AD and NAD patients across classes to be comparable between cohorts, the predicted percentage of $\mathrm{AD}$ patients in a given class depends itself on the prevalence of $\mathrm{AD}$ patients in the studied cohort. In the future, this scale might be improved by adding new variables and, in particular, the apolipoprotein E status, which today is not used routinely.

\section{Conclusion}

We developed a simple and intuitive prediction model that demonstrated its relevance in identifying groups of patients with different predictive values for AD. This new scale can be used to facilitate the interpretation and routine use of multivariate CSF data and to stratify patients in clinical research trials.

\section{Additional files}

Additional file 1: Cutoffs and logistic regression coefficients.

Additional file 2: Logistic regression and PLM scale classification.

\section{Abbreviations}

AD: Alzheimer disease; AUC: area under curve; $A \beta$ : amyloid $\beta ; C D R$ : clinical dementia rating; $\mathrm{Cl}$ : confidence interval; CSF: cerebrospinal fluid; 
ELISA: enzyme-linked immunosorbent assay; MCl: mild cognitive impairment; MRI: magnetic resonance imaging; NAD: non-Alzheimer disease; NINCDS-ADRDA: Institute of Neurological and Communicative Disorders and Stroke and the Alzheimer Disease and Related Disorders Association; NRI: Net Reclassification Index; PET: positron emission tomography; PLM: Paris-North, Lille, and Montpellier; QC: quality control; ROC: receiver operating characteristic.

\section{Competing interests}

All financial and material supports for this research are academic, and the authors declare no conflict of interest.

\section{Authors' contributions}

SL, JD, SS, CP, and AG: Study concept and design, analysis of the results, and drafting the manuscript. SL and JD: statistical analysis. SL, SS, KP, JLL, BJ, $\mathrm{MQM}, \mathrm{OB}, \mathrm{CMA}, \mathrm{NP}$, and BP: realization, follow-up and analysis of biologic measurements, and contribution to data interpretation and revision of the manuscript for important intellectual content. JD, DW, FB, EM, SB, DC, BC, DH, JH, JT, OV, CP, FP, and AG: clinical work, diagnosis confirmation, and contribution to data interpretation and revision of the manuscript for important intellectual content. All authors read and approved the final manuscript.

\section{Acknowledgements}

This work was supported through the National French Alzheimer effort (Plan Alzheimer) and the JPND program Biomark-APD. We thank Pr. Joe Menard for his very helpful advice. We thank Bénédicte Clément for editing the manuscript.

\section{Author details}

${ }^{1} \mathrm{CHU}$ de Montpellier and Université Montpellier I, IRMB, CCBHM, Laboratoire de Biochimie Protéomique Clinique, 80 Avenue Augustin Fliche, 34295 Montpellier, France. ${ }^{2}$ Centre Mémoire Ressources Recherche Paris Nord lle de France and Histologie et Biologie du Vieillissement, Groupe Hospitalier Saint-Louis Lariboisiere Fernand-Widal APHP, INSERM U942, Universite Paris Diderot, France. ${ }^{3}$ Inserm U837 and Neurobiology Unit, Centre de Biologie-Pathologie, CHU, Universite Lille Nord de France, 59045 Lille, France. ${ }^{4}$ Inserm U1079, University of Rouen, Department of Neurology and Laboratoire de biochimie, Rouen University Hospital, Rouen, France. ${ }^{5}$ Centre Mémoire Ressources Recherche, Alsace; Department of Neurology, University Hospital of Strasbourg, Strasbourg, France. ${ }^{6} 2$ ICube laboratory and FMTS (Fédération de Médecine Translationnelle de Strasbourg), team IMIS-Neurocrypto, University of Strasbourg and CNRS, Strasbourg, France. ${ }^{7}$ Centre Mémoire Ressources Recherche Besancon Franche-Comté, Department of Neurology, CHU Besançon, Besançon, France. ${ }^{8}$ Centre Mémoire Ressources Recherche, CHU, EA1040 Université Lille Nord de France, 59000 Lille, France. 'Laboratoire de Biochimie et de Biologie Moléculaire, Hôpital de Hautepierre, Hôpitaux Universitaire de Strasbourg, Strasbourg, France. ${ }^{10}$ Laboratoire de Biochimie Lariboisière-Fernand Widal Hospital, APHP, University Paris 7-Denis Diderot, University Paris Descartes, Paris, France. ${ }^{11}$ Laboratoire de Biochimie, CHU de Besançon, Besançon, France. ${ }^{12}$ Centre Mémoire Ressources Recherche Languedoc-Roussillon,

CHU de Montpellier, Hôpital Gui de Chauliac, Montpellier, and Université Montpellier I, Montpellier, France. ${ }^{13}$ Laboratoire de Neurosciences Cognitives et Adaptatives (LNCA), UMR7364, Université de Strasbourg-CNRS, Strasbourg, France.

\section{Received: 28 February 2014 Accepted: 13 June 2014}

Published: 26 June 2014

\section{References}

1. Dubois B, Feldman HH, Jacova C, Dekosky ST, Barberger-Gateau P, Cummings J, Delacourte A, Galasko D, Gauthier S, Jicha G, Meguro K, O'Brien J, Pasquier F, Robert P, Rossor M, Salloway S, Stern Y, Visser PJ, Scheltens P: Research criteria for the diagnosis of Alzheimer's disease: revising the NINCDS-ADRDA criteria. Lancet Neurol 2007, 6:734-746.

2. DeKosky ST, Carrillo MC, Phelps C, Knopman D, Petersen RC, Frank R, Schenk D, Masterman D, Siemers ER, Cedarbaum JM, Gold M, Miller DS, Morimoto $\mathrm{BH}$, Khachaturian AS, Mohs RC: Revision of the criteria for Alzheimer's disease: a symposium. Alzheimers Dement 2011, 7:e1-e12.

3. McKhann GM: Changing concepts of Alzheimer disease. JAMA 2011, 305:2458-2459.
4. Scheltens P, Erkinjunti T, Leys D, Wahlund LO, Inzitari D, del Ser T, Pasquier F, Barkhof F, Mantyla R, Bowler J, Wallin A, Ghika J, Fazekas F, Pantoni L: White matter changes on $C T$ and MRI: an overview of visual rating scales: European Task Force on Age-Related White Matter Changes. Eur Neurol 1998, 39:80-89.

5. Jack CR Jr, Wiste HJ, Vemuri P, Weigand SD, Senjem ML, Zeng G, Bernstein MA, Gunter JL, Pankratz VS, Aisen PS, Weiner MW, Petersen RC, Shaw LM, Trojanowski JQ, Knopman DS: Brain beta-amyloid measures and magnetic resonance imaging atrophy both predict time-to-progression from mild cognitive impairment to Alzheimer's disease. Brain 2011, 133:3336-3348.

6. Mouton-Liger F, Wallon D, Troussiere AC, Yatimi R, Dumurgier J, Magnin E, de la Sayette V, Duron E, Philippi N, Beaufils E, Gabelle A, Croisile B, Robert P, Pasquier F, Hannequin D, Hugon J, Paquet C: Impact of cerebro-spinal fluid biomarkers of Alzheimer's disease in clinical practice: a multicentric study. J Neurol 2014, 261:144-151.

7. Gabelle A, Dumurgier J, Vercruysse O, Paquet C, Bombois S, Laplanche JL, Peoc'h K, Schraen S, Buee L, Pasquier F, Hugon J, Touchon J, Lehmann S: Impact of the 2008-2012 French Alzheimer Plan on the use of cerebrospinal fluid biomarkers in research memory center: the PLM Study. J Alzheimers Dis 2013, 34:297-305.

8. Andreasen N, Minthon L, Davidsson P, Vanmechelen E, Vanderstichele $H$, Winblad B, Blennow K: Evaluation of CSF-tau and CSF-Abeta42 as diagnostic markers for Alzheimer disease in clinical practice. Arch Neurol 2001, 58:373-379

9. Blennow K, de Leon MJ, Zetterberg H: Alzheimer's disease. Lancet 2006, 368:387-403.

10. Shaw LM, Vanderstichele $H$, Knapik-Czajka M, Figurski M, Coart E, Blennow K, Soares H, Simon AJ, Lewczuk P, Dean RA, Siemers E, Potter W, Lee VM, Trojanowski JQ: Qualification of the analytical and clinical performance of CSF biomarker analyses in ADNI. Acta Neuropathol 2011, 121:597-609.

11. Tapiola T, Alafuzoff I, Herukka SK, Parkkinen L, Hartikainen P, Soininen H, Pirttila T: Cerebrospinal fluid \{beta\}-amyloid 42 and tau proteins as biomarkers of Alzheimer-type pathologic changes in the brain. Arch Neurol 2009, 66:382-389.

12. Lehmann S, Delaby C, Touchon J, Hirtz C, Gabelle A: Biomarkers of Alzheimer's disease: the present and the future. Rev Neurol (Paris) 2013, 169:719-723.

13. Roche S, Gabelle A, Lehmann S: Clinical proteomics of the cerebrospinal fluid: towards the discovery of new biomarkers. Proteomics 2008, 2:428-436.

14. Seshadri S, Fitzpatrick AL, Ikram MA, DeStefano AL, Gudnason V, Boada M, Bis JC, Smith AV, Carassquillo MM, Lambert JC, Harold D, Schrijvers EM, Ramirez-Lorca R, Debette S, Longstreth WT Jr, Janssens AC, Pankratz VS, Dartigues JF, Hollingworth P, Aspelund T, Hernandez I, Beiser A, Kuller LH, Koudstaal PJ, Dickson DW, Tzourio C, Abraham R, Antunez C, Du Y, Rotter II, et al: Genome-wide analysis of genetic loci associated with Alzheimer disease. JAMA 2010, 303:1832-1840.

15. Bjerke M, Portelius E, Minthon L, Wallin A, Anckarsater $H$, Anckarsater $\mathrm{R}$ Andreasen N, Zetterberg $H$, Andreasson U, Blennow K: Confounding factors influencing amyloid beta concentration in cerebrospinal fluid. Int $J$ Alzheimers Dis 2010, doi:10.4061/2010/986310.

16. Perret-Liaudet A, Pelpel M, Tholance $Y$, Dumont B, Vanderstichele H, Zorzi W, Elmoualij B, Schraen S, Moreaud O, Gabelle A, Thouvenot E, Thomas-Anterion C, Touchon J, Krolak-Salmon P, Kovacs GG, Coudreuse A, Quadrio I, Lehmann S: Risk of Alzheimer's disease biological misdiagnosis linked to cerebrospinal collection tubes. J Alzheimers Dis 2012, 31:13-20.

17. Lewczuk P, Beck G, Esselmann H, Bruckmoser R, Zimmermann R, Fiszer M, Bibl M, Maler JM, Kornhuber J, Wiltfang J: Effect of sample collection tubes on cerebrospinal fluid concentrations of tau proteins and amyloid beta peptides. Clin Chem 2006, 52:332-334.

18. Del Campo M, Mollenhauer B, Bertolotto A, Engelborghs S, Hampel H, Simonsen AH, Kapaki E, Kruse N, Le Bastard N, Lehmann S, Molinuevo JL Parnetti L, Perret-Liaudet A, Saez-Valero J, Saka E, Urbani A, Vanmechelen E, Verbeek M, Visser PJ, Teunissen C: Recommendations to standardize preanalytical confounding factors in Alzheimer's and Parkinson's disease cerebrospinal fluid biomarkers: an update. Biomark Med 2012, 6:419-430.

19. Mattsson N, Andreasson U, Persson S, Arai H, Batish SD, Bernardini S, Bocchio-Chiavetto L, Blankenstein MA, Carrillo MC, Chalbot S, Coart E, Chiasserini D, Cutler N, Dahlfors G, Duller S, Fagan AM, Forlenza O, Frisoni GB, Galasko D, Galimberti D, Hampel H, Handberg A, Heneka MT, Herskovits AZ, Herukka SK, Holtzman DM, Humpel C, Hyman BT, lqbal K, Jucker M, et al: The 
Alzheimer's Association external quality control program for cerebrospinal fluid biomarkers. Alzheimers Dement 2011, 7:386-395.

20. Lehmann S, Schraen S, Quadrio I, Paquet C, Bombois S, Delaby C, Dorey A Dumurgier J, Hirtz C, Krolak-Salmon P, Laplanche JL, Moreaud O, Peoc'h K, Rouaud O, Sablonniere B, Thouvenot E, Touchon J, Vercruysse O, Hugon J, Gabelle A, Pasquier F, Perret-Liaudet A: Impact of harmonization of collection tubes on Alzheimer's disease diagnosis. Alzheimers Dement 2013, doi:10.1016/j.jalz.2013.06.008.

21. Hulstaert F, Blennow K, Ivanoiu A, Schoonderwaldt HC, Riemenschneider M, De Deyn PP, Bancher C, Cras P, Wiltfang J, Mehta PD, lqbal K, Pottel H, Vanmechelen $E$, Vanderstichele $H$ : Improved discrimination of $A D$ patients using beta-amyloid(1-42) and tau levels in CSF. Neurology 1999, 52:1555-1562

22. de Jong D, Jansen RW, Kremer BP, Verbeek MM: Cerebrospinal fluid amyloid beta42/phosphorylated tau ratio discriminates between Alzheimer's disease and vascular dementia. J Gerontol A Biol Sci Med Sci 2006, 61:755-758.

23. Schoonenboom NS, Reesink FE, Verwey NA, Kester MI, Teunissen CE, van de Ven PM, Pijnenburg YA, Blankenstein MA, Rozemuller AJ, Scheltens P, van der Flier WM: Cerebrospinal fluid markers for differential dementia diagnosis in a large memory clinic cohort. Neurology 2012, 78:47-54.

24. Spies $P E$, Claassen JA, Peer PG, Blankenstein MA, Teunissen $C E$, Scheltens $P$, van der Flier WM, Olde Rikkert MG, Verbeek MM: A prediction model to calculate probability of Alzheimer's disease using cerebrospinal fluid biomarkers. Alzheimers Dement 2013, 9:262-268.

25. Dumurgier J, Vercruysse O, Paquet C, Bombois $\mathrm{S}$, Chaulet $\mathrm{C}$, Laplanche JL, Peoc'h K, Schraen S, Pasquier F, Touchon J, Hugon J, Lehmann S, Gabelle A: Intersite variability of CSF Alzheimer's disease biomarkers in clinical setting. Alzheimers Dement 2013, 9:406-413.

26. McKhann G, Drachman D, Folstein M, Katzman R, Price D, Stadlan EM: Clinical diagnosis of Alzheimer's disease: report of the NINCDS-ADRDA Work Group under the auspices of Department of Health and Human Services Task Force on Alzheimer's Disease. Neurology 1984, 34:939-944.

27. Perret-Liaudet A, Pelpel M, Tholance Y, Dumont B, Vanderstichele H, Zorzi W, El Moualij B, Schraen S, Moreaud O, Gabelle A, Thouvenot E, Thomas-Anterion C, Touchon J, Krolak-Salmon P, Kovacs GG, Coudreuse A, Quadrio I, Lehmann S: Cerebrospinal fluid collection tubes: a critical issue for Alzheimer disease diagnosis. Clin Chem 2012, 58:787-789.

28. Pencina MJ, D'Agostino RB Sr, D'Agostino RB Jr, Vasan RS: Evaluating the added predictive ability of a new marker: from area under the ROC curve to reclassification and beyond. Stat Med 2008, 27:157-172. discussion, 207-112.

29. Blennow K, Hampel H, Weiner M, Zetterberg H: Cerebrospinal fluid and plasma biomarkers in Alzheimer disease. Nat Rev Neurol 2010, 6:131-144

30. Morris JC: The Clinical Dementia Rating (CDR): current version and scoring rules. Neurology 1993, 43:2412-2414.

31. Scheltens P, Pasquier F, Weerts JG, Barkhof F, Leys D: Qualitative assessment of cerebral atrophy on MRI: inter- and intra-observer reproducibility in dementia and normal aging. Eur Neurol 1997, 37:95-99.

32. Prestia A, Caroli A, van der Flier WM, Ossenkoppele R, Van Berckel B, Barkhof F, Teunissen CE, Wall AE, Carter SF, Scholl M, Choo IH, Nordberg A, Scheltens P, Frisoni GB: Prediction of dementia in MCl patients based on core diagnostic markers for Alzheimer disease. Neurology 2013, 80:1048-1056.

doi:10.1186/alzrt267

Cite this article as: Lehmann et al:: A diagnostic scale for Alzheimer's disease based on cerebrospinal fluid biomarker profiles. Alzheimer's Research \& Therapy 2014 6:38.

\section{Submit your next manuscript to BioMed Central and take full advantage of:}

- Convenient online submission

- Thorough peer review

- No space constraints or color figure charges

- Immediate publication on acceptance

- Inclusion in PubMed, CAS, Scopus and Google Scholar

- Research which is freely available for redistribution

Submit your manuscript at www.biomedcentral.com/submit
C Biomed Central 